PROCEEDINGS OF THE

AMERICAN MATHEMATICAL SOCIETY

Volume 124, Number 4, April 1996

\title{
FREE PRODUCTS OF FINITARY LINEAR GROUPS
}

\author{
ORAZIO PUGLISI \\ (Communicated by Ron Solomon)
}

\begin{abstract}
In this note we show that the free product of any family of groups which are finitary linear over fields of the same characteristic $p \geq 0$, is still finitary linear over a field of characteristic $p$.
\end{abstract}

\section{INTRODUCTION}

Let $V$ be an $\mathbb{F}$-vector space, and $g$ an element of $\mathrm{GL}(V, \mathbb{F})$, the group of invertible $\mathbb{F}$-linear transformations of $V$. If $C_{V}(g)=\{v \mid v g=v\}$ has finite codimension in $V$ (or, equivalentely, if $[V, g]=\{v g-v \mid v \in V\}$ has finite dimension), the element $g$ is said to be a finitary linear transformation. When $V$ is a finite-dimensional vector space, then every element of $\mathrm{GL}(V, \mathbb{F})$ is finitary, so that this definition is of particular interest when $V$ has infinite dimension. It is easily seen that the finitary elements of $\mathrm{GL}(V, \mathbb{F})$ form a normal subgroup. This subgroup is denoted by $\operatorname{FGL}(V, \mathbb{F})$, and a group is said to be a finitary linear group if it is (isomorphic to) a subgroup of $\mathrm{FGL}(V, \mathbb{F})$ for some field $\mathbb{F}$ and $\mathbb{F}$-vector space $V$.

In recent years the structure of finitary groups has been the subject of intensive investigations which have led to a number of interesting results. The reader interested in the present situation in this area of research is referred to the papers of Hall and Phillips in $[\mathrm{A}]$ and to their bibliography.

Even though much is known about the structure of finitary linear groups, it seems to be a difficult question to decide which groups can have a finitary linear representation. It is very easy to show that any abelian group has a faithful finitary representation over a suitable field of characteristic zero, or that a group of the kind $H$ wr $K$, where $H$ is a finite dimensional linear group over $\mathbb{F}$ and $K$ is a group of finitary permutations, has a faithful representation over $\mathbb{F}$ (see $[\mathrm{P}]$ ), but, beyond that, very few results are available. It is worth mentioning that Meierfrankenfeld ([M]) has proved that a free group of infinite rank has an infinite-dimensional faithful finitary representation over any field of characteristic 0. Another remarkable result is contained in $[\mathrm{L}]$. A consequence of Theorem $\mathrm{D}$ of $[\mathrm{L}]$ is a necessary and sufficient condition for a countable FC $p$-group to be finitary linear over a field of characteristic $p$.

In this note we want to study how finitary linear groups behave with respect to free products. It turns out that their behaviour is reasonably good, since the following result holds:

Received by the editors October 5, 1994

1991 Mathematics Subject Classification. Primary 20E06, 20F29.

The author is a member of the G.N.S.A.G.A. 
Theorem 1. Let $G_{\lambda} \leq \operatorname{FGL}\left(V_{\lambda}, \mathbb{F}_{\lambda}\right), \lambda \in \Lambda$, be a family of finitary linear groups. If the fields $\mathbb{F}_{\lambda}$ have the same characteristic $p \geq 0$, then there exist a field $\mathbb{F}$ of characteristic $p$ and an $\mathbb{F}$-vector space $V$ such that the group $G=*_{\lambda \in \Lambda} G_{\lambda}$ has a faithful representation $\Theta: G \longrightarrow \operatorname{FGL}(V, \mathbb{F})$.

Actually, in the next section, a stronger form of this theorem will be proved.

Theorem 1 generalizes a well known result about free products of finite-dimensional linear groups; in fact, this generalization is obtained by combining the known result for finite-dimensional linear group with an argument involving ultraproducts.

Once we know that free products of finitary groups are still finitary, we might be interested in having more information about their representations. The analysis of a finitary representation of a free product gives the following result.

Theorem 2. Let $G=\boldsymbol{*}_{\lambda \in \Lambda} G_{\lambda}$ be a subgroup of $\operatorname{FGL}(V, \mathbb{F})$. If $|\Lambda| \geq 2$ and $G_{\lambda} \neq 1$ for all $\lambda \in \Lambda$, then any $G$-composition series of $V$ has at least one but finitely many factors on which $G$ acts faithfully, unless $G$ is the free product of two groups of order 2 . The action on a faithful factor is primitive provided the factor has infinite dimension.

When $G$ is the free product of two groups of order 2 then it is isomorphic to an infinite dihedral group. In this situation it is easy to see that a finitary $G$-module might not have any faithful composition section.

Finally, Theorem 2 is used to discuss the existence of an irreducible infinitedimensional finitary linear representation for a free product of finitary groups. As an application we show that a free group of infinite rank has a faithful and irreducible infinite-dimensional finitary representation over fields of any characteristic.

\section{THE PROOFS}

First of all we have to fix some notation and terminology.

If $g$ is an element of $\operatorname{GL}(V, \mathbb{F})$, we define the degree of $g$ as $\operatorname{deg}(g)=\operatorname{dim}([V, g])$. In the same way the degree of a subgroup $H \leq \mathrm{GL}(V, \mathbb{F})$ is defined as $\operatorname{deg}(H)=$ $\operatorname{dim}([V, K])$, where $[V, K]=\langle[V, k] \mid k \in K\rangle$. With this terminology the group $\operatorname{FGL}(V, \mathbb{F})$ turns out to be the subset of $\operatorname{GL}(V, \mathbb{F})$ consisting of the elements of finite degree.

When $\left\{G_{\lambda} \mid \lambda \in \Lambda\right\}$ is a family of groups, we indicate by $*_{\lambda \in \Lambda} G_{\lambda}$ their free product. Whenever the free product of the family $\left\{G_{\lambda} \mid \lambda \in \Lambda\right\}$ is considered, we agree that monomorphisms $\Gamma_{\lambda}: G_{\lambda} \longrightarrow \mathcal{*}_{\lambda \in \Lambda} G_{\lambda}$ have been chosen in such a way that

$$
\left\langle\left(G_{\lambda}\right) \Gamma_{\lambda} \mid \lambda \in \Lambda\right\rangle=\underset{\lambda \in \Lambda}{*} G_{\lambda} .
$$

If $H$ is any subgroup of $G_{\lambda}$, we shall write $\bar{H}$ instead of $(H) \Gamma_{\lambda}$.

If $\left\{G_{i} \mid i \in I\right\}$ is any family of groups, we shall indicate by $\operatorname{Dr}_{i \in I} G_{i}$ their direct product and by $\mathrm{Cr}_{i \in I} G_{i}$ their cartesian product. A survey of the basic properties of finitary linear groups can be found in $[\mathrm{P}]$.

We start by proving a simple fact which is a different version of Theorem 2.1 of $[\mathrm{H}]$.

Lemma 1. Let $G$ be a group and $\mathcal{H}=\left\{H_{\omega} \mid \omega \in \Omega\right\}$ a local system. For each $\omega \in \Omega$ let

$$
\rho_{\omega}: H_{\omega} \longrightarrow \operatorname{FGL}\left(V_{\omega}, \mathbb{F}_{\omega}\right)
$$


be a faithful finitary representation. If there exists a function $d: G \longrightarrow \mathbb{N}$ such that, if $x \in H_{\omega}$, then $\operatorname{deg}\left((x) \rho_{\omega}\right) \leq d(x)$, then $G$ has a faithful finitary representation

$$
\rho: G \longrightarrow \operatorname{FGL}(V, \mathbb{F}) \text {. }
$$

Moreover, if each $\mathbb{F}_{\omega}$ has characteristic $p$ ( $p$ zero or a positive prime ), also $\mathbb{F}$ can be chosen to have characteristic $p$.

Proof. Let $C=\mathrm{Cr}_{\omega \in \Omega} H_{\omega}$ and define the map

$$
\begin{aligned}
\alpha: G & \longrightarrow C, \\
g & \longmapsto\left(g_{\omega}\right)_{\omega \in \Omega},
\end{aligned}
$$

where $g_{\omega}=g$ if $g \in H_{\omega}$ and $g_{\omega}=1$ otherwise. In the appendix to section L of $[\mathrm{KW}]$, it is shown that an ultrafilter $\mathcal{U}$ on $\mathcal{H}$ can be chosen in such a way that the map

$$
\bar{\alpha}: G \longrightarrow\left(\mathrm{Cr}_{\omega \in \Omega} H_{\omega}\right) / \mathcal{U}
$$

induced by $\alpha$ is an injective homomorphism. Using the $\rho_{\omega}$ we can define an injective homomorphism

$$
\beta:\left(\mathrm{Cr}_{\omega \in \Omega} H_{\omega}\right) / \mathcal{U} \longrightarrow\left(\mathrm{Cr}_{\omega \in \Omega} \mathrm{GL}\left(V_{\omega}, \mathbb{F}_{\omega}\right)\right) / \mathcal{U}
$$

Set $\mathbb{F}=\left(\mathrm{Cr}_{\omega \in \Omega} \mathbb{F}_{\omega}\right) / \mathcal{U}$ and $V=\left(\mathrm{Cr}_{\omega \in \Omega} V_{\omega}\right) / \mathcal{U}$. Then $\left(\mathrm{Cr}_{\omega \in \Omega} \mathrm{GL}\left(V_{\omega}, \mathbb{F}_{\omega}\right)\right) / \mathcal{U}$ can be identified with $\mathrm{GL}(V, F)$ in a natural way.

The map

$$
\rho=\bar{\alpha} \beta: G \longrightarrow \mathrm{GL}(V, \mathbb{F})
$$

is an injective homomorphism. Fix any element $g \in G$. For each $\omega \in \Omega$ let $W_{g, \omega}$ be a subspace of $V_{\omega}$ such that

1. $\operatorname{dim}_{\mathbb{F}_{\omega}} W_{g, \omega} \leq d(g)$,

2. $\left[V_{\omega}, g_{\omega}\right] \leq W_{g, \omega}$.

Since $\left[\mathrm{Cr}_{\omega \in \Omega} V_{\omega}, g^{\alpha}\right] \leq \mathrm{Cr}_{\omega \in \Omega} W_{g, \omega}$, it is readily seen that

$$
[V,(g) \rho] \leq\left(\mathrm{Cr}_{\omega \in \Omega} W_{g, \omega}\right) / \mathcal{U} .
$$

But $\left(\mathrm{Cr}_{\omega \in \Omega} W_{g, \omega}\right) / \mathcal{U}$ has dimension at most $d(x)$ over $\mathbb{F}$, so that $\operatorname{deg}((g) \rho) \leq d(g)$ for every $g \in G$. This means that $(G) \rho \leq \operatorname{FGL}(V, \mathbb{F})$. To conclude the proof we have only to point out that, if the fields $\mathbb{F}_{\omega}$ have the same characteristic $p$, then also $\mathbb{F}$ has characteristic $p$.

The above lemma is not very useful, in general, since the existence of the function $d$ is actually a very strong hypothesis. On the other hand, in our setting, such a function will arise in a natural way.

Before going a step further we should point out a fact which will play an important role in the proof of our result.

Remark. Let $H_{i} \leq \operatorname{GL}\left(n_{i}, \mathbb{F}_{i}\right), i=1, \ldots, l$. If $n=\max \left\{n_{i} \mid i=1, \ldots, l\right\}$ and the $\mathbb{F}_{i}$ have the same characteristic, then, by 2.14 of $[\mathrm{W}], \boldsymbol{*}_{i=1}^{l} H_{i}$ has a faithful linear representation

$$
\psi: \underset{i=1}{\stackrel{\gtrless}{*}} H_{i} \longrightarrow \mathrm{GL}(n+1, \mathbb{F}),
$$

where $\mathbb{F}$ is a suitable field containing the fields $\mathbb{F}_{i}$. Moreover we can choose the embeddings $\Gamma_{\lambda}$ in such a way that, if $K \leq H_{i}$ for some $i$, we have $\operatorname{deg}(K)=$ $\operatorname{deg}((\bar{K}) \psi)$.

This is clear from the proof of 2.14 of [W].

At this stage another technical result is needed. 
Lemma 2. Let $H_{\lambda} \leq \mathrm{GL}\left(n_{\lambda}, \mathbb{F}_{\lambda}\right), \lambda \in \Lambda$, be a family of linear groups. If the fields $\mathbb{F}_{\lambda}$ have the same characteristic $p \geq 0$, then the free product of the $H_{\lambda}, \boldsymbol{*}_{\lambda \in \Lambda} H_{\lambda}$, has a faithful finitary representation

$$
\Theta: \underset{\lambda \in \Lambda}{*} H_{\lambda} \longrightarrow \operatorname{FGL}(V, \mathbb{F})
$$

over some field of characteristic $p$. Moreover, if $K \leq H_{\lambda}$, then $\operatorname{deg}((\bar{K}) \Theta)=$ $\operatorname{deg}(K)$.

Proof. For every finite subset $\Delta$ of $\Lambda$ we define $H_{\Delta}=\left\langle\overline{H_{\lambda}} \mid \lambda \in \Delta\right\rangle$. If

$$
\mathcal{F}=\{\Delta \subseteq \Lambda|| \Delta \mid<\infty\}
$$

then $\mathcal{L}=\left\{H_{\Delta} \mid \Delta \in \mathcal{F}\right\}$ is a local system for $H=\boldsymbol{*}_{\lambda \in \Lambda} H_{\lambda}$. For each $H_{\Delta} \in \mathcal{L}$ there is, by the preceding remark, a faithful representation

$$
\Theta_{\Delta}: H_{\Delta} \longrightarrow \operatorname{GL}\left(n_{\Delta}, \mathbb{F}_{\Delta}\right)
$$

such that $\operatorname{deg}\left((\bar{K}) \Theta_{\Delta}\right)=\operatorname{deg}(K)$ whenever $K$ is a subgroup of any $H_{\lambda}, \lambda \in \Delta$.

Now choose any element $x \in H$. By the uniqueness of the normal form, there exists a unique $\Delta \in \mathcal{F}$ such that $x \in H_{\Delta}$ and $x \notin H_{\Sigma}$ if $\Delta \nsubseteq \Sigma$. This means that, if $x \in H_{\Sigma}$, then $H_{\Delta} \leq H_{\Sigma}$. Now we want to bound $\operatorname{deg}\left((x) \Theta_{\Sigma}\right)$. Since $H_{\Delta} \leq H_{\Sigma}$, we have $\left(H_{\Delta}\right) \Theta_{\Sigma} \leq\left(H_{\Sigma}\right) \Theta_{\Sigma}$ and $(x) \Theta_{\Sigma} \in\left(H_{\Delta}\right) \Theta_{\Sigma}$. Thus $\operatorname{deg}\left((x) \Theta_{\Sigma}\right)$ can be bounded by

$$
\operatorname{deg}\left(\left(H_{\Delta}\right) \Theta_{\Sigma}\right) \leq \sum_{\lambda \in \Delta} \operatorname{deg}\left(\left(\overline{H_{\lambda}}\right) \Theta_{\Sigma}\right)=\sum_{\lambda \in \Delta} \operatorname{deg}\left(H_{\lambda}\right) .
$$

Define $d(x)=\sum_{\lambda \in \Delta} \operatorname{deg}\left(H_{\lambda}\right)$. Lemma 1 can now be applied to produce a finitary representation

$$
\Theta: H \longrightarrow \mathrm{FGL}(V, \mathbb{F})
$$

To conclude the proof we notice that, by the above remark, for every subgroup $K \leq H_{\lambda}$, the degree of $(\bar{K}) \Theta_{\Delta}$ is equal to $\operatorname{deg}(K)$ whenever $\lambda \in \Delta$. This implies that, in the ultraproduct representation, the degree of $(\bar{K}) \Theta$ is still equal to $\operatorname{deg}(K)$.

A straightforward consequence is

Corollary 1. In the notation of Lemma 2, if the set $\left.\left\{\operatorname{deg}\left(H_{\lambda}\right)\right) \mid \lambda \in \Lambda\right\}$ is not bounded, then $\operatorname{deg}\left(\left(\boldsymbol{*}_{\lambda \in \Lambda} G_{\lambda}\right) \Theta\right)$ is infinite.

Proof. This is clearly true, $\operatorname{since} \operatorname{deg}\left(\left(\boldsymbol{*}_{\lambda \in \Lambda} G_{\lambda}\right) \Theta\right) \geq \operatorname{deg}\left(\overline{G_{\lambda}}\right)=\operatorname{deg}\left(G_{\lambda}\right)$ for each $\lambda \in \Lambda$.

This corollary shows, for instance, that the free product of any infinite family of finite groups is an infinite dimensional finitary linear group in any characteristic. In fact, if $\mathcal{G}=\left\{G_{i} \mid i \in I\right\}$ is such a family, choose any field $\mathbb{F}$ and an unbounded set $\left\{n_{i} \in \mathbb{N} \mid i \in I\right\}$. For each $n_{i}$ we can find a faithful representation for $G_{i}$ over $\mathbb{F}$ with $\operatorname{deg}\left(G_{i}\right) \geq n_{i}$. Now we apply Lemma 2 and Corollary 1 to show that $\boldsymbol{*}_{i \in I} G_{i}$ is finitary linear of infinite dimension.

We are now in a position to prove our main result. As announced in the introduction, a stronger version of Theorem 1 can be proved. 
Theorem A. Let $G_{\lambda} \leq \operatorname{FGL}\left(V_{\lambda}, \mathbb{F}_{\lambda}\right), \lambda \in \Lambda$, be a family of finitary linear groups. If the fields $\mathbb{F}_{\lambda}$ have the same characteristic $p \geq 0$, then there exist a field $\mathbb{F}$ of characteristic $p$ and an $\mathbb{F}$-vector space $V$ such that the group $G=*_{\lambda \in \Lambda} G_{\lambda}$ has a faithful representation

$$
\Theta: G \longrightarrow \mathrm{FGL}(V, \mathbb{F}) .
$$

Moreover, if $K$ is any finitely generated subgroup of $G_{\lambda}$, then $\operatorname{deg}((\bar{K}) \Theta)=\operatorname{deg}(K)$. Proof. Define $\mathcal{F}_{\lambda}=\left\{F \leq G_{\lambda} \mid F\right.$ is finitely generated $\}$. The set

$$
\mathcal{H}=\left\{\left\langle\overline{F_{\lambda}} \mid \lambda \in \Lambda\right\rangle \mid F_{\lambda} \in \mathcal{F}_{\lambda} \lambda \in \Lambda\right\}
$$

is a local system for $G$. For each element $H$ of $\mathcal{H}$, Lemma 2 affords a faithful finitary representation

$$
\Theta_{H}: H \longrightarrow \mathrm{FGL}\left(V_{H}, \mathbb{F}_{H}\right),
$$

where the degree of any element $x$ can be bounded by a function $d(x)$ which does not depend on $H$. Moreover, if $K \leq F_{\lambda} \in \mathcal{F}_{\lambda}$, $\operatorname{deg}\left((\bar{K}) \Theta_{H}\right)=\operatorname{deg}(K)$. Now Lemma 1 applies to give the claim.

Now we can say something about the representations of free products. First of all, we need a simple fact about free products which is certainly well known. We prefer, anyway, to give its proof.

Lemma 3. Let $G=*_{\lambda \in \Lambda} G_{\lambda}$, where $|\Lambda|>1$ and $G_{\lambda} \neq 1 \forall \lambda \in \Lambda$. If $N, M$ are non-trivial normal subgroups, then $N \cap M \neq 1$.

Proof. Since $N, M$ are normal in $G$, they contain elements which are not in any conjugate of the free factors of $G$. Pick $x \in N, y \in M$ satisfying this property. If $N \cap M=1$ then $[N, M]=1$, so that $[x, y]=1$. By 4.1.6 of [MKS], $x$ and $y$ should be powers of a certain element $z$. Since $x, y$ are not contained in any conjugate of the free factors, their order cannot be finite. Hence $\langle x\rangle \cap\langle y\rangle \neq 1$. This is false since $N \cap M=1$.

We have enough information to begin the study of a general finitary representation of a free product. From now on $G=\boldsymbol{*}_{\lambda \in \Lambda} G_{\lambda}$ will be a subgroup of $\operatorname{FGL}(V, \mathbb{F})$. To avoid trivial cases we assume, moreover, that $\Lambda$ contains at least 2 elements and that the groups $G_{\lambda}$ are non-trivial.

Proof of Theorem 2. Choose any $G$-composition series in $V$ and let $\left\{V_{i} \mid i \in I\right\}$ be the set of its non-trivial composition factors. If we indicate by $G_{i}$ the group induced by $G$ on $V_{i}$ we have, by Lemma 13 of $[\mathrm{P}]$, that $G / U(G)$ is a subdirect product of the $G_{i}$. Here $U(G)$ stands for the maximal normal unipotent subgroup of $G$. Since $U(G)$ is contained in the Hirsch-Plotkin radical of $G$, we have that $U(G)=1$. This is true because $|\Lambda|>1$ and $G_{\lambda} \neq 1 \forall \lambda \in \Lambda$, and $G$ is not the free product of two cyclic groups of order 2. Thus $G \leq \operatorname{Dr}_{i \in I} G_{i}$. Call $\pi_{i}$ the projection on the $i$-th component. Set $A=\left\{i \in I \mid \operatorname{ker}\left(\pi_{i}\right)=1\right\}$ and $B=\complement_{I} A$. The action of $G$ on $V_{i}$ is faithful if and only if $i \in A$. Suppose that $A$ is empty, so that $U(G)=\bigcap_{i \in B} \operatorname{ker}\left(\pi_{i}\right)=1$, and choose any element $g \in G$ which is not contained in any conjugate of the free factors. Let $S(g)=\left\{i \mid(g) \pi_{i} \neq 1\right\}$. By our choice $g$ is an element of infinite order, and, since $g$ is a finitary transformation, the set $S(g)$ is finite. By Lemma 3 the normal subgroup

$$
K=\bigcap_{i \in S(g)} \operatorname{ker}\left(\pi_{i}\right)
$$


is non-trivial, so that we can choose $1 \neq k \in K$. Thus $[g, k]=1$ and, again by 4.1.6 of [MKS], $g$ and $k$ should be powers of the same element $h$. This is impossible since $g$ has infinite order and $\langle g\rangle \cap\langle k\rangle=1$. Thus $A$ must contain at least one element.

Now we show that $A$ is finite. Of course $G$ is a subdirect product of the $G_{i}, i \in A$. If $\rho: G \longrightarrow \operatorname{Dr}_{i \in A} G_{i}$ is the injection of $G$ into $\operatorname{Dr}_{i \in A} G_{i}$, then $(x) \rho$ has only finitely many non-trivial components. On the other hand, since the action of $G$ on each of the $G_{i}, i \in A$, is faithful, the $i$-th component of $(x) \rho(i \in A)$ is trivial if and only if $x=1$. This proves that $A$ is finite.

To prove the final part of the claim, assume that $V$ is an infinite-dimensional irreducible $G$-module. If the action of $G$ on $V$ is imprimitive, then $G$ possesses a normal subgroup $N$ which is the subdirect product of infinitely many finite dimensional linear groups, and $G / N$ is a transitive group of finitary permutations. As an $N$-module, $V$ is the direct sum of finite dimensional submodules $\left\{V_{i} \mid i \in I\right\}$, which are permuted transitively by $G / N$. Now choose an element $g \in N$ such that $g$ is not contained in any conjugate of any free factor. Since the set $J=\left\{i \in I \mid\left[V_{i}, g\right] \neq 0\right\}$ is finite there is, by Lemma 2.3 of $[\mathrm{N}]$, an $x \in G$ such that

$$
\left\{V_{i} \mid i \in J\right\} \cap\left\{V_{i} \mid i \in J\right\} x=\emptyset .
$$

A consequence of this fact is that $\langle g\rangle \cap\left\langle g^{x}\right\rangle=1$. Moreover, since (*) holds, $\left[g, g^{x}\right]=1$, and this is impossible because $g$ and $g^{x}$ are not in a conjugate of a free factor nor are they powers of the same element.

This theorem has an easy corollary.

Corollary 2. Let $G_{\lambda} \leq \operatorname{FGL}\left(V_{\lambda}, \mathbb{F}_{\lambda}\right), \lambda \in \Lambda$, be a family of finitary linear groups, where the fields $\mathbb{F}_{\lambda}$ have the same characteristic $p \geq 0$. If $|\Lambda|>1$ and at least one member of the family has no finite dimensional linear representations in characteristic $p$, then $G=*_{\lambda \in \Lambda} G_{\lambda}$ has a faithful and irreducible finitary representation over a field of characteristic $p$.

Proof. Let $V$ be any finitary module in characteristic $p$ for $G=*_{\lambda \in \Lambda} G_{\lambda}$. By Theorem 2, $V$ has at least one irreducible and faithful $G$-factor $W=T / B$. Since at least one of the free factors, say $G_{\alpha}$, has no finite dimensional faithful linear representations in characteristic $p$, it follows that $W$ must have infinite dimension. $\square$

More generally, under the same hypothesis of the above corollary, the free product $G=*_{\lambda \in \Lambda} G_{\lambda}$ has a faithful and irreducible finitary representation of infinite dimension over a field of characteristic $p$ whenever, for some reason, there does not exist a finite dimensional linear group (in characteristic $p$ ) containing copies of the groups $G_{\lambda}$. We now discuss one of these cases.

As an application of Theorem 2 we want to prove that a free group of infinite rank $\mu$ has an infinite-dimensional faithful finitary linear representation over fields of any characteristic.

When the characteristic is 0 , this result was proved to be true in $[\mathrm{M}]$. So choose a positive prime $p$ and let $I$ be any infinite set of cardinality $\mu$. For every $i \in I$ let $G_{i}$ be a finite elementary abelian $q$-group of rank $n_{i}$ for some prime $q \neq p$. Choose the $n_{i}$ in such a way that the set $\left\{n_{i} \mid i \in I\right\}$ is unbounded. For each $i \in I, G_{i}$ has a faithful finite-dimensional representation over $\mathbb{F}_{p}$, the field with $p$ elements. Now consider the finitary representation afforded by Theorem A for the group $G=*_{i \in I} G_{i}$. The field $\mathbb{F}$ still has characteristic $p$. Let $W=T / B$ be 
a faithful $G$-composition section of $V$. If $W$ has finite dimension, say $n$, then all the abelian $q$-subgroups of $G$ should have rank bounded by $n$, and this is not the case by our choice of the $G_{i}$. So $W$ has infinite dimension. Since every free factor $G_{i}$ can be mapped isomorphically onto the $i$-th component of $\operatorname{Dr}_{i \in I} G_{i}$, there is an homomorphism of $G$ onto $\operatorname{Dr}_{i \in I} G_{i}$, whose kernel $K$ is a free group. To show this recall that, by the Kuroš Subgroup Theorem (see [R], 6.3.1), $K$ is a free product of the form $H *\left(*_{t \in T}\left(K \cap G_{i}^{t}\right)\right)$, where $H$ is a free group and $T$ a suitable subset of $G$. Since $K \cap G_{i}=1 \forall i \in I$, also $K \cap G_{i}^{g}=1 \forall i \in I$ and $g \in G$. Thus $K$ is a free group. It is readily seen that the rank of $K$ is $\max \left\{\aleph_{0},|I|\right\}=|I|=\mu$. We can now use the results contained in $[\mathrm{M}]$ to show that $W$ has an irreducible $K$ submodule $U \neq 0$. Since the set $\{U g \mid g \in G\}$ would be a system of imprimitivity for $G$ in $W$, it turns out that $U=W$, so that $K$ acts faithfully and irreducibly on $W$. Hence every free group on infinite rank has a faithful and irreducible finitary representation over some field of characteristic $p$, thus proving our claim.

\section{REFERENCES}

[A] B. Hartley, G. M. Seitz, A. V. Borovik, and R. M. Bryant (eds.), Finite and locally finite groups, NATO ASI Series C471, Kluwer Academic Publishers, Dordrecht, Boston, and London, 1995.

$[\mathrm{H}]$ J. Hall, Finitary linear transformation groups and elements of finite local degree, Arch. Math. (Basel) 50 (1988), 315-318. MR 89b:20067

[KW] O. H. Kegel and B. A. F. Wehrfritz, Locally finite groups, North Holland, Amsterdam London, 1973. MR 57:9848

[L] F. Leinen, Hypercentral unipotent finitary skew linear groups, Comm. Algebra 22 (1994), 929-949. MR 95d:20087

$[\mathrm{M}] \quad$ U. Meierfrankenfeld, Ascending subgroups of irreducible finitary linear groups, J. London Math. Soc. (2) 51 (1995), 75-92. CMP 95:6

[MKS] W. Magnus, A. Karrass and D. Solitar, Combinatorial group theory, Dover, New York, 1976. MR 54:10423

[N] P. M. Neumann, The structure of finitary permutation groups, Arch. Math. (Basel) 27 (1976), 3-17. MR 53:5754

[P] R. E. Phillips, The structure of groups of finitary transformations, J. Algebra 119 (1988), 400-448. MR 90a:20080

[R] D. J. S. Robinson, A course in the theory of groups, Springer Verlag, Berlin - Heidelberg - New York, 1982. MR 84k:20001

[W] B. A. F. Wehrfritz, Infinite linear groups, Spinger, Berlin - Heidelberg - New York, 1973. MR 49:436

Dipartimento di Matematica, Università degli Studi di Trento, I-38050 Povo (Trento), Italy

E-mail address: puglisi@alpha.science.unitn.it 